\title{
Integrierte Versorgung für Borderline-Patienten - ein längst überfälliges Konzept
}

\author{
Integrated Care for Patients with Borderline Personality Disorder - \\ A Long Overdue Concept
}

Autoren

Institut
Andreas Schindler, Martin Lambert, Anne Karow, Jürgen Gallinat, Ingo Schäfer

Klinik für Psychiatrie und Psychotherapie, Universitätsklinikum Hamburg-Eppendorf
Bibliografie

DOI http://dx.doi.org/

10.1055/s-0042-116651

Psychiat Prax 2016; 43:

409-410

(c) Georg Thieme Verlag KG

Stuttgart $\cdot$ New York

ISSN 0303-4259

\section{Korrespondenzadresse}

Dr. Andreas Schindler

Klinik für Psychiatrie und

Psychotherapie, Universitäts-

klinikum Hamburg-Eppendorf

Martinistraße 52

20246 Hamburg

a.schindler@uke.de
Die Borderline-Persönlichkeitsstörung (BPS) ist eine komplexe Störung, die zu erheblichem Leid bei Betroffenen und Angehörigen führen kann. In etwa einem Drittel der Fälle erfüllt die BPS die Kriterien einer „schweren psychischen Erkrankung“" (SMI) [1]. Der Anteil der BPS an allen schwer psychisch Kranken lässt sich auf etwa 11\% schätzen [2]. BPS-Patienten beanspruchen das Hilfesystem überproportional stark und verursachen mit 15\% der Behandlungsfälle 25\% der Gesamtkosten stationär psychiatrischer Behandlungen [3]. Eine neuere Analyse [4] kommt auf gesamtgesellschaftliche Kosten von durchschnittlich $26882 €$ pro Patient und Jahr. Die Hälfte dieser Kosten entfällt auf stationär psychiatrische Behandlungen. Hiervon wiederum entfallen aber nur 30\% auf störungsspezifische stationäre Psychotherapie (die die Kosten in den Folgejahren deutlich senkt) aber 70\% auf Kriseninterventionen.

Das zu diesen Daten passende klinische Szenario beinhaltet oft, dass sich Patientinnen und Patienten mit BPS in einer akuten Krisensituation notfallmäßig in die Klinik begeben und aufgrund von Selbstgefährdung auf eine Akutstation aufgenommen werden. Je weniger diese auf die interaktionellen Eigenheiten von BPS-Patienten eingestellt ist, desto größer ist die Gefahr einer weiteren Eskalation und desto weniger kann therapeutisch gearbeitet werden. Die Patienten werden nach Stabilisierung oft ohne adäquate ambulante Weiterbehandlung wieder nach Hause entlassen und das Szenario wiederholt sich in der nächsten Krise.

Voraussetzung für eine Reduktion solcher Krisen, für eine nachhaltige Stabilisierung der Patientinnen und Patienten und damit auch für eine Reduktion der Krankheitskosten ist ein langfristig und kontinuierlich strukturiertes Behandlungsangebot. Dieses muss Kriseninterventionen ermöglichen, ambulante und stationäre Settings integrieren und störungsspezifisch angelegt sein.
Zwar liegen mehrere wirksame psychotherapeutische Behandlungsmodelle vor [5], von langfristig und kontinuierlich angelegten, störungsspezifischen Behandlungsstrukturen kann in der Versorgungslandschaft der BPS aber leider bisher kaum die Rede sein.

Zwar ist das Bild aufgrund fehlender Versorgungsforschung bruchstückhaft, es weist aber auf massive quantitative und qualitative Unzulänglichkeiten hin. An spezialisierten (teil-)stationären Kapazitäten gibt es bundesweit etwa 700 Behandlungsplätze. Selbst bei einer konservativ geschätzten Punktprävalenz von 0,7\% der 82 Mio. Bundesbürger ist das sehr wenig und erklärt die langen Wartezeiten [6]. Wie die Situation in psychiatrischen Kliniken allgemein aussieht, lässt sich gegenwärtig nicht überblicken. Auf eine Umfrage [7] aus dem Jahr 2009 antworteten überhaupt nur $1 / 4$ der deutschen Kliniken. Von diesen gaben immerhin $3 / 4$ an, spezialisierte stationäre Angebote vorzuhalten. Spezialisierte ambulante Behandlungsplätze sind nochmals erheblich seltener als stationäre [6]. Leider liegen bislang keine belastbaren Zahlen zu spezifischen BPS-Behandlungsangeboten in psychiatrischen Institutsambulanzen (PIA) vor. PiAs sind ein wichtiger Baustein der ambulanten Behandlung und potenziell eine zentrale Schnittstelle zwischen stationären und ambulanten Angeboten. Allerdings setzt deren Finanzierung der Behandlung schwer kranker, „betreuungsintensiver“ Patienten enge Grenzen. Im niedergelassenen Bereich nimmt der Anteil störungsspezifisch ausgebildeter Psychotherapeuten und Psychiater zwar zu, die strukturellen Rahmenbedingungen erschweren hier die Behandlung schwer kranker BPS-Patienten aber noch stärker als im Bereich der PIAs. Ein weiteres Problem ist die mangelnde Vernetzung der verschiedenen Behandlungsbausteine. In einigen Regionen haben sich Netzwerke gebildet, von einer flächendeckenden Vernetzung sind wir aber noch weit entfernt [8]. 
Der zentrale Behandlungsbaustein, der am ehesten eine langfristig angelegte, störungsspezifische Behandlung schwer kranker BPS-Patienten verspricht, fehlt aber bis heute völlig: die „Integrierte Versorgung“ (IV). Diese wird bereits in den S2-Behandlungsleitlinien der BPS von 2009 [5] gefordert, ist aber mit Ausnahme der 2014 angelaufenen Hamburger IV-Borderline bislang nicht umgesetzt [9]. Einige weitere Projekte unter dem Label „IV“, weisen bei weitem nicht die nach $\S 140$ SGB geforderten und im funktionalen Basismodell ausformulierten Strukturen auf [10]. Zwei amerikanische Pilotstudien zeigen, dass störungsspezifische IV-Strukturen zu einer Verbesserung von Lebenszufriedenheit und beruflicher Funktionsfähigkeit sowie zu einer deutlichen Reduktion von stationären Behandlungstagen führen $[11,12]$. Wenig erfolgreich war dagegen der Versuch, BPS-Patienten im Rahmen von Projekten für Psychosepatienten mit zu behandeln [13]. Zu unterschiedlich sind Problematik, Interaktionsmuster und Hilfesuchverhalten.

Wie könnte eine störungsspezifische und strukturell angemessene IV-Borderline aussehen? Unseres Erachtens liegen mit der dialektisch-behavioralen Therapie (DBT) [14] als therapeutischem Basiskonzept und mit den Strukturen der Integrierten Versorgung [15] die notwendigen Bausteine vor. Ein multiprofessionelles, ambulantes IV-Kernteam gewährleistet eine langfristige, intensive und bei Bedarf nachgehende Komplexbehandlung. Dieses Team bietet ein umfassendes Case Management, die multimodale psychotherapeutische und psychiatrische Behandlung sowie abgestufte Möglichkeiten der Krisenintervention. Es kann außerdem auf die ambulanten, stationären und teilstationären Strukturen eines psychiatrischen Krankenhauses mit regionalem Versorgungsauftrag zurückgreifen. Das IV-Kernteam „dosiert“ die Intensität und Frequenz seine Leistungen flexibel und angepasst an den gegenwärtigen Hilfebedarf des Patienten. Inhaltlich erarbeiten Behandler und Patient zunächst ein verlässliches, akzeptierendes und veränderungsorientiertes Therapiebündnis. Auf dieser Basis werden dann frühzeitig die Fähigkeiten zur Selbstregulation gestärkt und detailliert ein Vorgehen in Krisensituationen entwickelt. Übergeordnetes Therapieziel ist die Reduktion dysfunktionalen und krisengenerierenden sowie die Förderung funktionalen Verhaltens. Darüber hinaus werden die Behandlungsziele individuell mit den Patienten erarbeitet. Obwohl die DBT für einen psychotherapeutischen Ansatz bereits sehr umfangreich konzipiert ist, stellt das Konzept einer IV-Borderline eine erhebliche strukturelle Erweiterung dar, vor allem in Hinblick auf mögliche Behandlungssettings, sozialpsychiatrische und über die DBT hinausgehende psychotherapeutische Elemente. Im Vergleich mit bisherigen sozialpsychiatrischen Angeboten stellt es eine Erweiterung im Sinne einer dringend notwendigen störungsspezifischen Behandlung der BPS dar.
Vor dem Hintergrund der durch die BPS entstehenden Belastungen bei Betroffenen und Angehörigen und angesichts der strukturellen Unzulänglichkeiten der BPS-Behandlung erscheint uns das Konzept der IV-Borderline längst überfällig.

\section{Literatur}

1 Delespaul P, Consensusgroep EPA. Consensus regarding the definition of persons with severe mental illness and the number of such persons in the Netherlands. Tijdschrift voor psychiatrie 2013; 55: 427-438

2 Lora A, Bezzi R, Erlicher A. Estimating the prevalence of severe mental illness in mental health services in Lombardy (Italy). Community Mental Health Journal 2007; 43: 341-357

3 Bohus M. Zur Versorgungssituation von Borderline-Patienten in Deutschland. Persönlichkeitsstörungen: Theorie und Therapie 2007; 11: $149-153$

4 Wagner T, Roepke S, Marschall P et al. Krankheitskosten der Borderline Persönlichkeitsstörung aus gesellschaftlicher Perspektive. Zeitschrift für Klinische Psychologie und Psychotherapie 2013; 42: 242 - 255

5 Bohus M, Buchheim P, Doering $S$ et al. S2 Praxisleitlinien in Psychiatrie und Psychotherapie. Band 1: Behandlungsleitlinie Persönlichkeitsstörungen. Heidelberg: Steinkopff; 2009

6 Richter C, Steinacher B, Eschenhoff $A$ et al. Stationäre und teilstationäre DBT-Angebote für Patienten mit Borderline-Persönlichkeitsstörung Ergebnisse einer deutschlandweiten Umfrage. Verhaltenstherapie 2014; 24: 265-271

7 Jacob GA, Allemann R, Schornstein K et al. Zur aktuellen Situation der stationären Versorgung von Patienten mit Borderline-Persönlichkeitsstörung in Deutschland. Psychiat Prax 2009; 36: 387-389

8 Gunia H. Psychotherapie von Menschen mit einer Borderline-Persönlichkeitsstörung in einem ambulanten Netzwerk. Psychotherapie im Dialog 2014; 3: 60-63

9 Schmid P, Steinert T, Borbé R. Systematische Literaturübersicht zur Implementierung der sektorübergreifenden Versorgung (Regionalbudget, integrierte Versorgung) in Deutschland. Psychiat Prax 2013; 40: $414-424$

10 Steinhart I, Wienberg G. Das funktionale Basismodell für die gemeindepsychiatrische Versorgung schwer psychisch kranker Menschen Mindeststandards für Behandlung und Teilhabe. Psychiat Prax 2016; 43: $65-68$

11 Cunningham $K$, Wolbert $R$, Lillie $B$. It's about me solving my problems: Clients' assessments of Dialectical Behavior Therapy. Cognitive and Behavioral Practice 2004; 11: 248-256

12 American Psychiatric Association. Gold Award. Integrating dialectical behavioral therapy into a community mental health program. Psychiatric Services 1998; 49: 1338-1340

13 Horvitz-Lennon M, Reynolds S, Wolbert $R$ et al. The role of Assertive Community Treatment in the treatment of people with Borderline Personality Disorder. American Journal of Psychiatric Rehabilitation 2009; 12: $261-277$

14 Linehan MM. DBT Skills Training Manual. New York: Guilford Press; 2014

15 Lambert M, Bock T, Meigel-Schleiff $C$ et al. Integrierte Versorgung von Patienten mit psychotischen Erkrankungen nach dem Hamburger Modell: Rationalen, Behandlungsmodell und Ergebnisse der Vorstudie. Psychiat Prax 2014; 41: 257-265 